\title{
Radiological-prognostic correlation of diffusion tensor imaging in a mild traumatic brain injury model
}

\author{
YUTING ZHANG ${ }^{1-3 *}$, LUSHENG $\mathrm{LI}^{2-4 *}$ and LING HE ${ }^{1-3}$ \\ ${ }^{1}$ Department of Radiology, Children's Hospital of Chongqing Medical University; \\ ${ }^{2}$ Key Laboratory of Pediatrics in Chongqing; ${ }^{3}$ Ministry of Education Key Laboratory of Child Development and Disorders; \\ ${ }^{4}$ Department of Neurosurgery, Children's Hospital of Chongqing Medical University, Chongqing 400014, P.R. China
}

Received February 07, 2020; Accepted September 10, 2020

DOI: $10.3892 / \mathrm{etm} .2020 .9386$

\begin{abstract}
Diffusion tensor imaging (DTI) parameters can detect changes in the brain microstructure in mild traumatic brain injury (mTBI). Whether these parameter changes can predict neural functional recovery after mTBI is still relatively unknown. The present study aimed to investigate the radiological-prognostic correlation between these radiological parameters and learning and memory deficits using an in-house constructed rat model of mTBI. We established a rat model of diffuse axonal injury (DAI) at different injury levels, followed by magnetic resonance imaging at 6,24 , and $72 \mathrm{~h}$, and 1, 2 weeks post injury, and randomly selected the rats for analysis of histopathology and learning and memory deficits. DTI parameters and $\beta$-amyloid precursor protein ( $\beta$-APP) levels were obtained to estimate the extent of brain injury and the correlation with the times of crossing the safety platform as measured using a water maze test. The results revealed that fractional anisotropy (FA) was sensitive to axonal integrity. FA values of the corpus callosum in the injury groups decreased at all time points post injury, except in the mild injury group, which recovered to normal levels at 1 and 2 weeks post-injury. The neural function of the mild injury group recovered to normal compared with the normal control group. FA value, $\beta$-APP of corpus callosum in different groups at $24 \mathrm{~h}$ post injury showed obvious correlation with learning and memory deficits at the recovery stage $(r=0.881, r=-0.931)$. In conclusion, DTI can reflect varying injury states of DAI over time with direct comparison to histopathology and could be used to predict the neural functional recovery at the early stage post-injury in a rat model.
\end{abstract}

Correspondence to: Professor Ling He, Department of Radiology, Children's Hospital of Chongqing Medical University, 136 Zhongshand 2nd Road, Chongqing 400014, P.R. China

E-mail: doctorheling@yeah.net

*Contributed equally

Key words: mild traumatic brain injury, diffuse axonal injury, diffusion tensor imaging, rat model, neural functional recovery

\section{Introduction}

Mild traumatic brain injury (mTBI) is a growing public health concern globally, representing $70-90 \%$ of all TBIs (1). Traumatic brain injury resulting from impactacceleration forces leads to diffuse axonal injury (DAI), which is considered to be a key feature of pathology following mTBI. mTBI is difficult to detect on conventional computed tomography (CT) or magnetic resonance imaging (MRI) (2). While most patients with mTBI symptoms recover in 7-10 days, $10-40 \%$ of patients may suffer persistent symptoms, with long-term cognitive deficits and white matter changes (3). Furthermore, repeated mTBI may cause an increased incidence of chronic traumatic encephalopathy (4). However, it is difficult to predict the prognosis of neural functional recovery after DAI at the early stage, and clinically relevant experimental models of DAI still require improvement (5). Consequently, we simulated the DAI occurrence mechanism with reference to Namjoshi et al (6) and Li et al (7) injury device and constructed an in-house rat model of DAI, which enabled simultaneous and instantaneous extra-large rotational acceleration and linear acceleration at the head of the rat, thus providing a useful tool for DAI study (8).

The diffusion of water molecules is closely related to changes to the myelin sheath, axolemma, and ultra-structure inside the axon after axonal injury. Diffusion tensor imaging (DTI) can detect the diffusion changes in water molecules and the coherence of fibrous structures sensitively in patients with DAI (9). DTI can assess the random movement of protons of water molecules in terms of the apparent diffusion coefficient (ADC) and orientational dependence (fractional anisotropy, FA). The corpus callosum is a common site of high tension from impact, with frequent abnormalities identified by DTI $(10,11)$. Axonal injury has been characterized histologically using $\beta$-amyloid precursor protein ( $\beta$-APP) immunohistochemistry (IHC), which normally traverses the length of the axon, and accumulates in response to injury (12). The aims of this study were to determine the diagnostic accuracy of DTI in a rat model of DAI with direct comparison to histopathologically-confirmed axonal injury, and to identify any correlations with neural functional recovery. 


\section{Materials and methods}

DAI model establishment. All studies were approved by the Animal Ethics Committee of Chongqing Medical University (approval no. 20170301). One hundred adult male Sprague-Dawley rats, aged 2-3 months and weighing $300-400 \mathrm{~g}$, were used in this study. The animals were kept in cages at room temperature of $22-25^{\circ} \mathrm{C}$, air humidity of $40 \%$, air pressure of $101.325 \mathrm{kPa}$ and $12 \mathrm{~h}$ light/dark cycles. All animals had free access to common rat feed and water. The rats were randomly allocated to two groups (control, $\mathrm{n}=25$; experimental, $\mathrm{n}=75$ ). The self-made injury device was used to establish the DAI rat model at different injury levels. The device comprises a pneumatic conversion device driven by a high air flow rate. The air pressure of the power cylinder reaches the preset air pressure $(0.8 \mathrm{kPa})$ through the charging of the electric air charging pump. The pneumatic conversion system is started by pushing the gear slider to make a linear acceleration movement to the right in an instant. With the action of the slider, the gears rotate and move around its center at the same time via the meshing action of the gears and racks to produce the compound motion of instantaneous super linear acceleration and angular acceleration. The start switch of the pneumatic transfer system is triggered continuously, which can realize multiple round-trip rotation and acceleration. According to the number of repeated injuries delivered, the rats were divided into the mild injury group $\mathrm{A}$, the moderate injury group $\mathrm{B}$, and the severe injury group $\mathrm{C}$, with 25 rats in each group (please refer to the schematic diagram shown in Fig. 1).

All the rats of the experimental group were anesthetized via an intraperitoneal injection of chloral hydrate $(300 \mathrm{mg} / \mathrm{kg})$. The head of the rat in the experimental group was fixed in the rat connector. The actuating cylinder was inflated to $0.8 \mathrm{kPa}$. The rats in group A, B, and C received repeated axial linear plus sagittal rotational acceleration impact for 1,2, and 3 times, respectively, through the swinging lever and rotating part. The injured rats were monitored post-injury until they awakened and could move normally. The wake-up time and recovery time interval of neurophysiological reflexes (corneal reflex, hindlimb claw reflex, and righting reflex) in the injured groups were observed.

Methods of rat euthanasia. Humane endpoints were established following the University of Pennsylvania's Institutional Animal Care and Use Committee (IACUC) guideline in order to minimize pain or distress to experimental animals. Cervical vertebra dislocation was used for euthanasia, as rapid brain tissue collection is required for neurophysiological studies. The rats were anesthetized via an intraperitoneal injection of chloral hydrate $(300 \mathrm{mg} / \mathrm{kg})$ prior to euthanasia by cervical vertebra dislocation. Cardiorespiratory arrest was used to verify rat death.

Magnetic resonance imaging. At 6, 24, 72 h, and 1, 2 weeks post-injury, to prevent the influence on the DTI scanning result caused by limb exercise, unstable breath, and heart rate, 10 of the 25 rats in each group (control group and experimental group A, B, C) received intraperitoneal anesthetization with chloral hydrate $(300 \mathrm{mg} / \mathrm{kg}$ ). An MRI scanner (Philips Achieva 3.0T) and an 8-channel knee-phased-array coil were used for MRI.
Turbo spin-echo T2-weighted image parameters were as follows: Repetition time (TR) $=5,000 \mathrm{msec}$; echo time $(\mathrm{TE})=100 \mathrm{msec}$; slice thickness $=2.0 \mathrm{~mm}$; field of view $=74 \times 74 \times 42 \mathrm{~mm}^{3}$; and voxel size $=0.3 \times 0.3 \times 2.0 \mathrm{~mm}^{3}$. DTI data were acquired using a spin echo planar sequence with $\mathrm{TR} / \mathrm{TE}=2907 \mathrm{msec} / 63 \mathrm{msec} ; \mathrm{b} 0=0 \mathrm{sec} / \mathrm{mm}^{2} ; \mathrm{b} 1=1,000 \mathrm{sec} / \mathrm{mm}^{2}$; direction number $=25$, slice thickness $=2.0 \mathrm{~mm}$; number of signal averages $(\mathrm{NSA})=5$; and field of view $(\mathrm{FOV})=74 \times 74 \times 42 \mathrm{~mm}$.

Post-image processing. Post-image processing was acquired at the Extended MR WorkSpace workstation (version no. 2.6.3.5; Philips Medical Systems Mr, Inc.) with the production of an FA map, an ADC map and a 3D tensor tractography (3D-DTT) map. Since the corpus callosum and external capsule of the rat have a continuous white board layer, the dividing line between the two was unified and defined as the following, according to brain stereotaxic atlas: Inferior margin ligature of cingulate cortex (bregma + 1.60-0.92 mm), inferior margin ligature of fimbria hippocampi (bregma-0.92-2.80 mm) and inferior margin ligature of hippocampi (bregma-2.80-5.30 mm) (13). The dividing line for the corpus callosum and external capsule was the transverse ligature of the inferior margin of hippocampi, based on which the external capsule was divided into the left part and the right part (Fig. 2).

One radiologist with 8 years of neural imaging experience, who was blinded to the experimental grouping, carefully delineated the regions of interest (ROIs) of the corpus callosum, left and right internal capsule, external capsule, and the pyramidal tract, to acquire the FA and ADC values on the DTI image. To increase the accuracy of the measured values, T2-weighted image (T2WI) images were first merged with the corresponding parameter maps, and then used to demonstrate the margins of the freehand ROIs.

$I H C$. At 6, 24 and $72 \mathrm{~h}$, and 1 and 2 weeks post-injury, 3 rat brains from each group from the remaining 15 rat brains were extracted and fixed in $4 \%$ paraformaldehyde for $48 \mathrm{~h}$ at room temperature. Following this, the brains were cryosectioned at $5 \mu \mathrm{m}$ for $\beta$-APP IHC analysis, according to previously published protocol (14). The IHC data were quantified independently by two investigators from the corresponding locations matching to the MRI data for correlation analysis via the threshold of the normal staining intensity as determined by the control staining. IHC quantification by each investigator was averaged to represent the percentage of positive $\beta$-APP staining in the images (magnification, $\mathrm{x} 400$ ).

Morris water maze test. Morris water maze tests were performed at 2 weeks post-injury to test learning and memory deficits in the rats. The Morris water maze was comprised of a video monitoring system, round water pool and safety platform. The safety platform was at the 3rd quadrant. The water temperature was $23-26^{\circ} \mathrm{C}$. The video monitoring system was used to film and record the incubation period (time required to locate the platform) and track by which the rat locates the safety platform. The rats were trained for 8 days. The first day was used to get the rats accustomed to the water environment without a safety platform. During the following 6 days, the water level was raised by $1 \mathrm{~cm}$ to test each rat for 4 times to locate the platform, with each test lasting $60 \mathrm{sec}$. If the rat failed to locate the platform, the incubation period was 
Table I. Behavioral observation of rats of each group $(n=25$, mean $\pm S D)$.

\begin{tabular}{lccrr}
\hline Recovery time (min) & Group A & Group B & Group C & P-value \\
\hline Corneal reflex & $28.50 \pm 5.72$ & $39.50 \pm 4.79^{\mathrm{a}}$ & $44.80 \pm 2.35^{\mathrm{a}, \mathrm{b}}$ & $<0.05$ \\
Hind paw reflex & $42.80 \pm 4.50$ & $52.80 \pm 8.27^{\mathrm{a}}$ & $69.80 \pm 11.08^{\mathrm{a}, \mathrm{b}}$ & $<0.05$ \\
Righting reflex & $58.10 \pm 7.14$ & $80.80 \pm 11.16^{\mathrm{a}}$ & $106.20 \pm 16.23^{\mathrm{a}, \mathrm{b}}$ & $<0.05$ \\
Wake-up time & $56.84 \pm 8.43$ & $76.54 \pm 10.85^{\mathrm{a}}$ & $101.57 \pm 15.56^{\mathrm{a}, \mathrm{b}}$ & $<0.05$
\end{tabular}

${ }^{\mathrm{a}} \mathrm{P}<0.05$, compared to group $\mathrm{A} ;{ }^{\mathrm{b}} \mathrm{P}<0.05$, compared to group $\mathrm{B}$.

recorded as $60 \mathrm{sec}$. The test on day 7 was conducted with the removal of the safety platform and testing for $60 \mathrm{sec}$. The first 3 days comprised the training period and days 4-7 were used to measure the incubation period while the rats were trying to locate the safety platform. These were tests of the positioning and navigation ability. On day 8 , the safety platform was removed and the times that the rats succeeded in crossing the safety platform within $60 \mathrm{sec}$ were recorded. Furthermore, the percentage of searching time for the safety platform quadrant was recorded, which was a test of space exploring ability.

Statistical analysis. Statistical analysis was conducted by using SPSS for Windows software (version no. 20.0; IBM, Corp.). Data are presented as mean \pm standard deviation. One-way ANOVA followed by Bonferroni's correction was used for multiple comparisons. The correlation between two variables was assessed by Pearson correlation analysis. Enumeration data are presented as a percentage and compared using the $\chi^{2}$ test. $\mathrm{P}<0.05$ was considered to indicate a statistically significant difference.

\section{Results}

Behavioral observation of rats in the acute stage of injury. After injury, the rats developed limb convulsions, incontinence, and apnea in varying degrees. Corneal reflex, hind paw reflex, and righting reflex disappeared in all three injury groups. The average recovery time of corneal reflex, hind paw reflex, and righting reflex, and the wake-up time were significantly different between group A and group B, and between group B and group $\mathrm{C}(\mathrm{P}<0.05$; Table $\mathrm{I})$. After waking, the rats showed decreased activity, slow reaction, and unsteady walking.

DTI image and diffusion parameter analysis. There were no obvious changes in the signal intensity or gray gradient in the T2WI, FA map, and ADC map of the corpus callosum, bilateral internal capsule, external capsule, and pyramidal tract of each group.

Quantitative analysis of diffusion parameters showed that FA values of the corpus callosum in each injury group decreased from $6 \mathrm{~h}$ post-injury, reached the lowest level at $24 \mathrm{~h}$ post-injury $(\mathrm{P}<0.05)$, and then started to recover. FA values of the corpus callosum in the mild injury group A recovered to normal level at 1 and 2 weeks post-injury, but did not recover in group B and C (Fig. 3A). FA and ADC values of the bilateral external capsule, internal capsule, and pyramidal tract in each injury group showed no significant differences with those of the control group (Table SI).

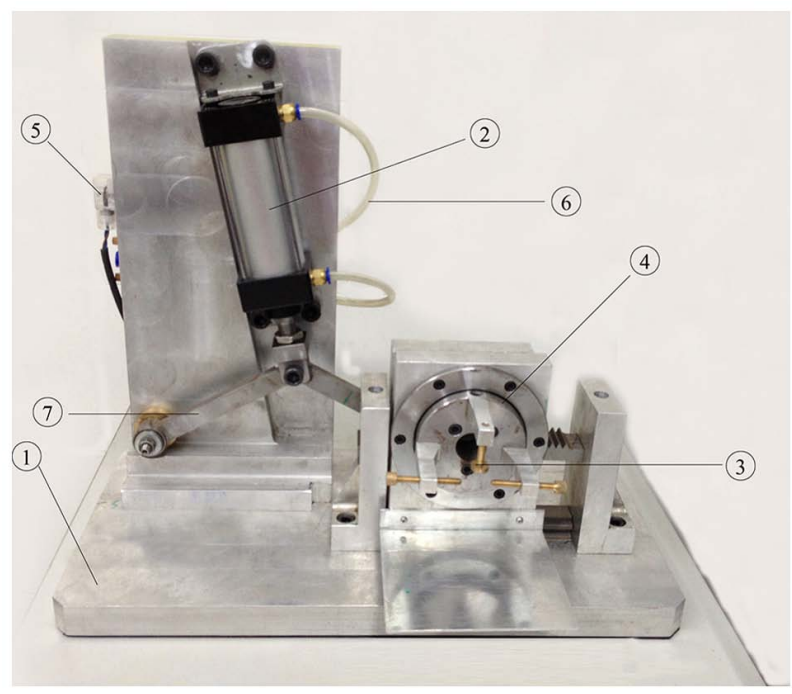

Figure 1. Schematic diagram of the DAI device. 1, pedestal; 2, actuating cylinder; 3 , the rat connector; 4 , rotating part; 5 , solenoid valve; 6 , breather pipe; and 7, swinging lever. DAI, diffuse axonal injury.

IHC results. IHC staining in the corpus callosum of the injury groups demonstrated changes consistent with white matter damage (Fig. 4). The percentage of positive $\beta$-APP staining in the corpus callosum of group $\mathrm{A}, \mathrm{B}$, and $\mathrm{C}$ at each time point post-injury increased compared with that of the control group, and there was a significant difference between each injury group. The percentage of positive $\beta$-APP staining increased from $6 \mathrm{~h}$ post-injury, reached the highest level at $24 \mathrm{~h}$ post-injury, and then started to recover. The rats in group A recovered normal $\beta$-APP levels at 2 weeks post-injury (Fig. 3B and Table II).

Correlation between $\beta$-APP and FA values of the corpus callosum. The correlations between the $\beta$-APP and FA values of the corpus callosum in group A, B, and C at 24 h post-injury were analyzed. The results showed an obvious negative correlation, with an $r$ value of -0.723 .

\section{Result of the Morris water maze test.}

Test of positioning and navigation ability. From the test of positioning and navigation ability, it was found that in the 6-day training period, the incubation period (time required to find the platform) of the rats in each group decreased as the training time increased. The incubation period of group B and $\mathrm{C}$ was longer than that of the control group $(\mathrm{P}<0.05)$, whereas the difference between group $\mathrm{A}$ and the control group was not statistically 
A

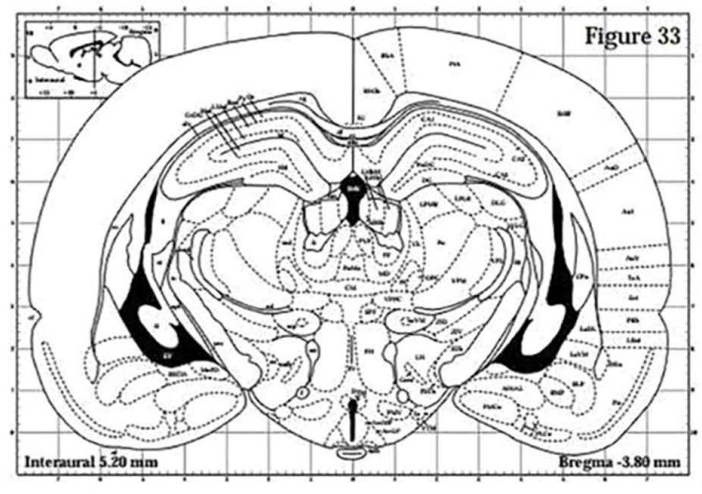

C

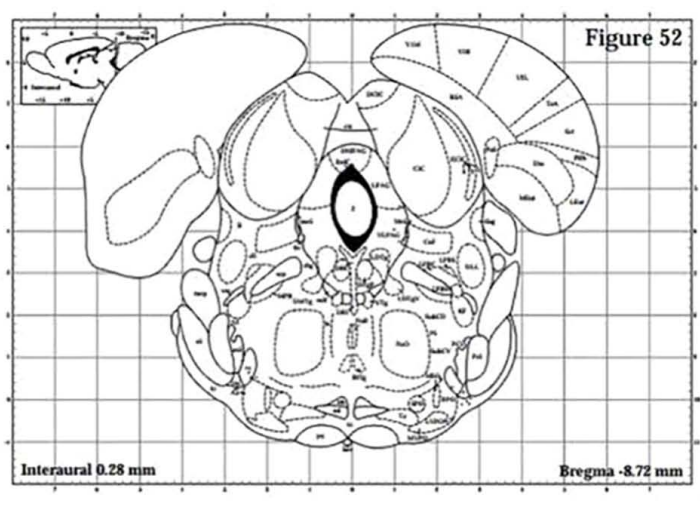

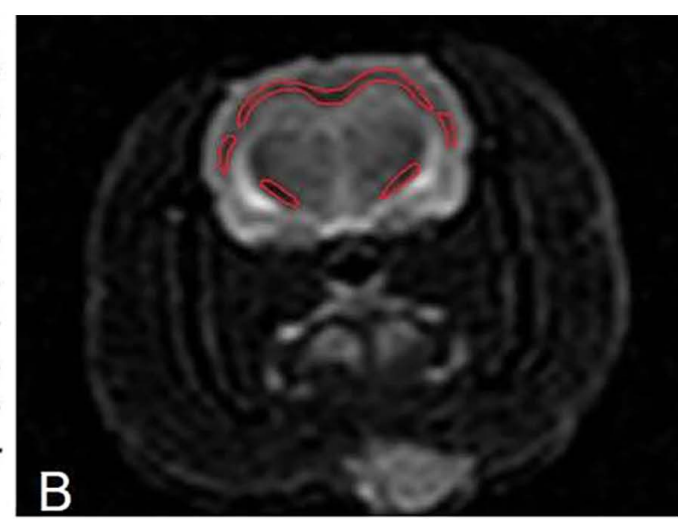

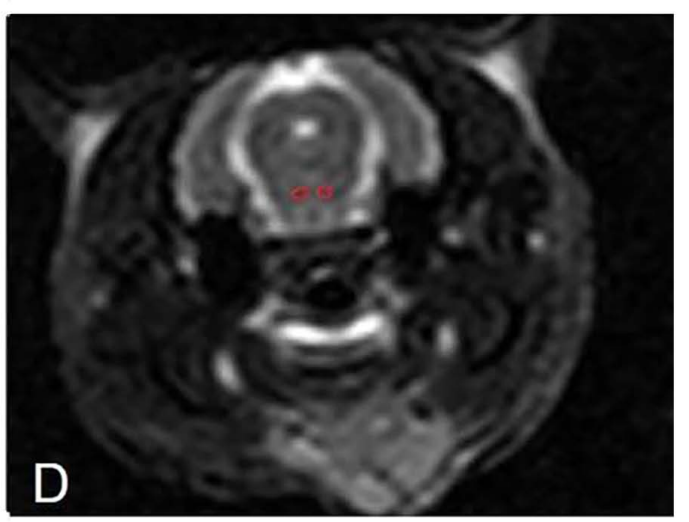

Figure 2. (A) Rat brain stereotaxic atlas for the corpus callosum, internal capsule, and external capsule. (B) The structures shown in MRI. (C) Rat brain stereotaxic atlas for the pyramidal tract. (D) The structures shown in MRI.

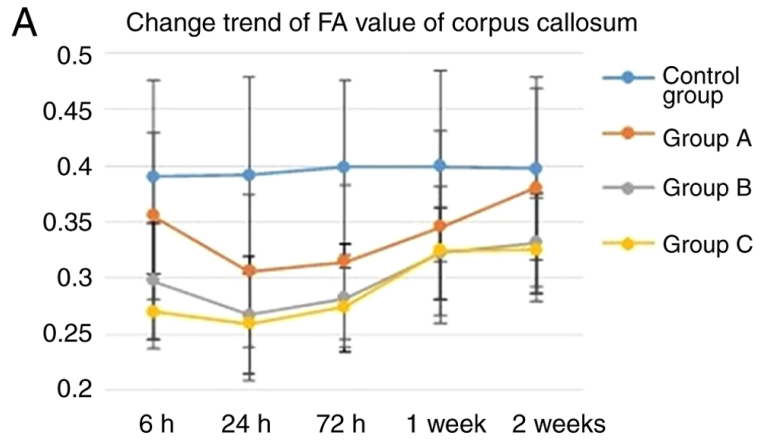

B 40 Change trend of $\beta$-APP of corpus callosum

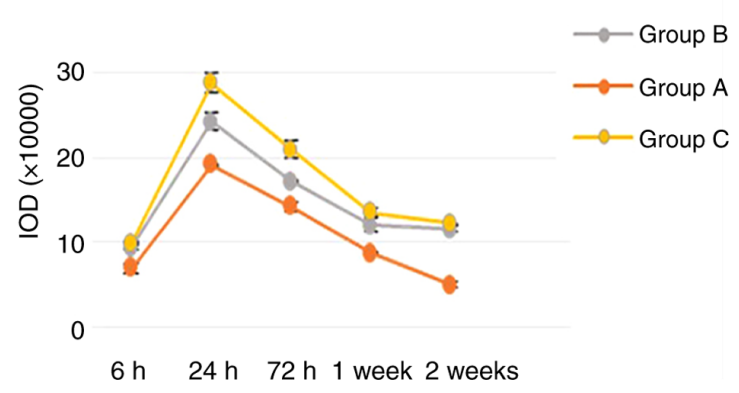

Figure 3. (A) Change trend of the FA value of the corpus callosum in each group at each time point. (B) Change trend of the $\beta$-APP level in the corpus callosum in each group at each time point. FA, fractional anisotropy; $\beta$-APP, $\beta$-amyloid precursor protein.

significant $(\mathrm{P}>0.05)$. The differences among groups $\mathrm{A}, \mathrm{B}$, and $\mathrm{C}$ showed statistical significance $(\mathrm{P}<0.05$; Table SII).

Test of space exploration ability. In the test of space exploration ability, the platform searching ability of groups $\mathrm{B}$ and $\mathrm{C}$ was poorer compared with that of the control group $(\mathrm{P}<0.05)$, whereas the difference between group $\mathrm{A}$ and the control group was not statistically significant $(\mathrm{P}>0.05)$. The difference among group $\mathrm{A}, \mathrm{B}$, and $\mathrm{C}$ showed statistical significance $(\mathrm{P}<0.05)$. The times of crossing safety platform of the control group and group A, B, C were 6.60 $\pm 0.89,6.20 \pm 1.09,3.33 \pm 0.89$, and $1.67 \pm 0.82$, respectively (Table SIII).

Trajectory chart analysis. During the training period, the rats in the control group firstly sailed along the pool wall and then along the diagonal line of the pool. Their motion track was mostly a straight line. The injured rats spent longer time to sail along the pool wall. In addition, the track that the rats used to look for the platform was mostly curved (Fig. S1).

Correlation analysis for FA, $\beta$-APP and learning and memory deficits of the rats during the recovery stage. Correlations among the FA, $\beta$-APP staining of the corpus callosum at $24 \mathrm{~h}$ post injury and the results of crossing the safety platform at 2 weeks post injury in group A, B, and C were analyzed. The result showed an obvious positive correlation with an $r$ value of 0.881 between the FA and the results of crossing the safety platform (Fig. S2A), and obvious negative correlation with an $\mathrm{r}$ value of -0.931 between the $\beta$-APP staining and the results of crossing the safety platform (Fig. S2B). 
Table II. The percentage of positive $\beta$-APP staining of each group $(n=3$, mean \pm SD).

\begin{tabular}{|c|c|c|c|c|}
\hline Time points & Control group & Group A & Group B & Group C \\
\hline $6 \mathrm{~h}$ & $4.31 \pm 0.19$ & $6.92 \pm 0.58^{a}$ & $9.34 \pm 0.25^{\mathrm{a}, \mathrm{b}}$ & $9.86 \pm 0.17^{\mathrm{a}, \mathrm{b}}$ \\
\hline $24 \mathrm{~h}$ & $4.54 \pm 0.17$ & $19.18 \pm 0.11^{\mathrm{a}}$ & $24.29 \pm 1.00^{\mathrm{a}, \mathrm{b}}$ & $28.70 \pm 1.21^{\mathrm{a}-\mathrm{c}}$ \\
\hline $72 \mathrm{~h}$ & $4.44 \pm 0.38$ & $14.16 \pm 1.53^{\mathrm{a}}$ & $17.18 \pm 0.15^{\mathrm{a}, \mathrm{b}}$ & $20.92 \pm 0.98^{\mathrm{a}-\mathrm{c}}$ \\
\hline 1 week & $4.50 \pm 0.26$ & $8.65 \pm 0.10^{\mathrm{a}}$ & $12.03 \pm 0.74^{\mathrm{a}, \mathrm{b}}$ & $13.50 \pm 0.45^{\mathrm{a}-\mathrm{c}}$ \\
\hline 2 week & $4.68 \pm 0.31$ & $4.97 \pm 0.34$ & $11.52 \pm 0.33^{\mathrm{a}, \mathrm{b}}$ & $12.24 \pm 0.02^{\mathrm{a}-\mathrm{c}}$ \\
\hline
\end{tabular}

${ }^{a} \mathrm{P}<0.05$, compared to the control group; ${ }^{\mathrm{b}} \mathrm{P}<0.05$, compared to group $\mathrm{A} ;{ }^{\mathrm{c}} \mathrm{P}<0.05$, compared to group $\mathrm{B}$. $\beta$-APP, $\beta$-amyloid precursor protein.

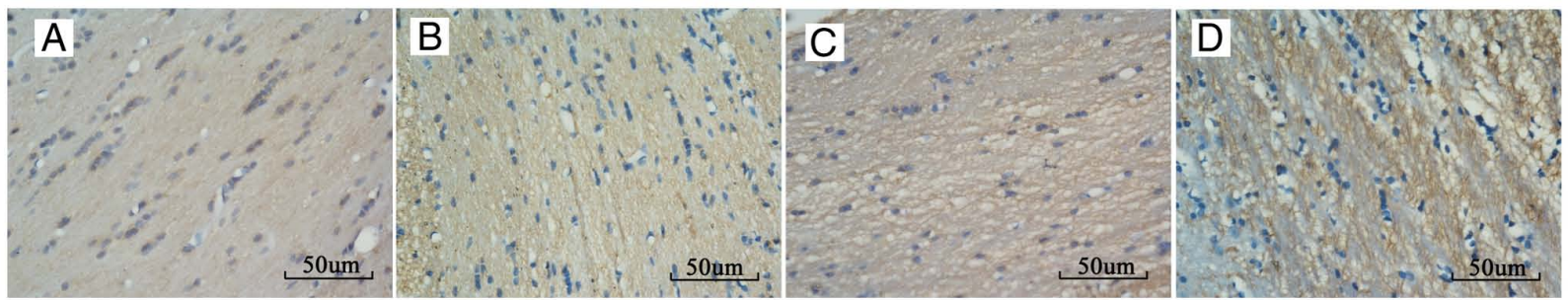

Figure 4. The positive $\beta$-APP staining intensity in the corpus callosum of each group (A) Control group; (B) mild injury group A; (C) moderate injury group B; (D) severe injury group C. $\beta$-APP, $\beta$-amyloid precursor protein.

\section{Discussion}

Diffuse axonal injury (DAI) resulting from impact-acceleration forces, including linear, rotational, or some combination, is a common cause of substantial neurological impairment. The pathologies following DAI including primary axonal damage and secondary degeneration, remain insufficiently characterized (15). Compared with widely used experimental models of mild traumatic brain injury (mTBI), such us fluid percussion (FP) and controlled cortical impact (CCI) systems, and closed head injury (CHI) models, various other models have been developed to increase rotational acceleration, which are more clinically relevant to human head movement following impact-related Mtbi $(5,6,14)$. We simulated the DAI occurrence mechanism with reference to Namjoshi et al (6) and Li et al (7) injury devices and constructed an in-house rat model of DAI, which enabled simultaneous and instantaneous extra-large sagittal rotational acceleration and axial linear acceleration at the head of the rat, with direct comparison to histopathologically-confirmed axonal injury, as assessed by $\beta$-amyloid precursor protein ( $\beta$-APP), which normally traverses the length of the axon and accumulates in response to injury (12). We demonstrated previously that this model integrates histopathological and behavioral characteristics of DAI, without focal injuries, such as skull fracture, hemorrhage, and surgery $(8,16)$.

In the present study, the percentage of positive $\beta$-APP staining in the corpus callosum of group A, B, and C at each time point post-injury increased compared with that in the control group, and there was a significant difference between each injury group, which demonstrated that the extent of DAI that incurred using our in-house rat model was directly proportional to the severity of injury.

DAI is usually irregular, without hemorrhage, and is difficult to detect on conventional CT or MRI, and mainly relies on patient self-reporting for clinical diagnosis (17). Diffusion tensor imaging (DTI) can detect the diffusion changes of water molecules and the coherence of fibrous structures in patients with DAI, which provides important information in vivo (9). The fractional anisotropy (FA) value reflects the anisotropy of water molecule diffusion, and is sensitive to axonal integrity. The apparent diffusion coefficient (ADC) reflects the water molecule diffusion characteristics, influenced by cellular brain edema (CBE) (18). The results of the present study showed that the FA value could reflect varying axonal injury states over time, and was obviously correlated with the change in $\beta$-APP levels in the corpus callosum. The more severe the axonal injury was, the higher $\beta$-APP value and the lower FA value would be. As an in vivo radiological parameter, the FA value might replace $\beta$-APP, the early molecular marker of DAI injury, to reflect the degree of early axonal injury.

Cognitive and behavioral deficits caused by DAI may persist for months to years (19). Axonal injury of the brain centerline and neural pathway are closely related to learning and memory deficits (20). Kraus et al (3) and Lipton et al (21) reported that DTI might determine the relationship between cognitive deficits and TBI. However, it is difficult to predict the prognosis of neural functional recovery after DAI at the early stage. We detected significant differences in learning and memory deficits between the injury groups, which demonstrated that the more severe the injury the rats suffered, the worse were their learning and memory deficits. Furthermore, an obvious correlation between the FA value of the corpus callosum at the early stage and learning and memory deficits of the rats at the recovery stage were found, and both the FA value and the learning and memory deficits of the rats in the mild injury group could recover to normal levels after 2 weeks of follow up. This suggested that DTI could predict the recovery of the rat's learning and memory deficits at an early stage post-injury.

The present study had some limitations. First, the magnetic field intensity of the 3T MRI machine is relatively low, making 
it difficult to obtain a clear image of small animals. Additionally, if different pathology components coexist in one image voxel, because of the partial volume effects, DTI measurement would be affected, making accurate estimation of the diffusion parameters difficult (22). Further evaluation of axonal injury, demyelination, axonal loss, and inflammation with improved parametric diffusion methods will be of interest to determine the overall water molecule diffusion features of the white matter after DAI.

In conclusion, DTI provided complementary insight into the underlying pathologies reflecting varying injury states over time, and could be used to predict the neural functional recovery at the early stage post-injury in a DAI rat model. We propose DTI as a sensitive quantification method for early-stage diagnosis and prognostic analysis of DAI.

\section{Acknowledgements}

The authors would like to thank Dr Xianyong Tang (College of Mechanical Engineering, Chongqing University), and Dr Jin Zhu (Department of Pathology, Children's Hospital of Chongqing Medical University), for the experimental guidance of immunohistochemistry.

\section{Funding}

No funding was received.

\section{Availability of data and materials}

The datasets used and/or analyzed during the current study are available from the corresponding author on reasonable request.

\section{Authors' contributions}

LL and LH conceived and designed the study. YZ and LL performed the experiments. YZ, LL and LH wrote the manuscript. All authors read and approved the final manuscript.

\section{Ethics approval and consent to participate}

The current study were approved by the Animal Ethics Committee of Chongqing Medical University (approval no. 20170301).

\section{Patient consent for publication}

Not applicable.

\section{Competing interests}

The authors declare that they have no competing interests.

\section{References}

1. Cassidy J, Carroll L, Peloso P, Borg J, von Holst H, Holm L, Kraus J and Coronado V; WHO Collaborating Centre Task Force on Mild Traumatic Brain Injury: Incidence, risk factors and prevention of mild traumatic brain injury: Results of the WHO collaborating Centre task force on mild traumatic brain injury. J Rehabil Med 36 (Suppl 43): S28-S60, 2004.
2. Smith DH, Meaney DF and Shull WH: Diffuse axonal injury in head trauma. J Head Trauma Rehabil 18: 307-316, 2003.

3. Kraus MF, Susmaras T, Caughlin BP, Walker CJ, Sweeney JA and Little DM: White matter integrity and cognition in chronic traumatic brain injury: A diffusion tensor imaging study. Brain 130: 2508-2519, 2007.

4. McKee AC, Stern RA, Nowinski CJ, Stein TD, Alvarez VE, Daneshvar DH, Lee HS, Wojtowicz SM, Hall G, Baugh CM, et al: The spectrum of disease in chronic traumatic encephalopathy. Brain 136: 43-64, 2013.

5. Xiong Y, Mahmood A and Chopp M: Animal models of traumatic brain injury. Nat Rev Neurosci 14: 128-142, 2013.

6. Namjoshi DR, Cheng WH, McInnes KA, Martens KM Carr M, Wilkinson A, Fan J, Robert J, Hayat A, Cripton PA and Wellington CL: Merging pathology with biomechanics using chimera (closed-head impact model of engineered rotational acceleration): A novel, Surgery-free model of traumatic brain injury. Mol Neurodegen 9: 55, 2014.

7. Li XY, Dai GH, Feng DF, Li J and Gu L: Experimental device for inducing diffuse brain injury in small animals. BME Clin Med 13: 489-493, 2009.

8. Xiang L, Zhang YT, Liang P, Wei H, Peng LL and Li LS: Study on the establishment of rat model of different degree of diffuse axonal injury. Chongqing Med 45: 2881-2884, 2016.

9. Mayer AR, Ling J, Mannell MV, Gasparovic C, Phillips JP, Doezema D, Reichard R and Yeo RA: A prospective diffusion tensor imaging study in mild traumatic brain injury. Neurology 74: 643-650, 2010.

10. McAllister TW, Ford JC, Ji S, Beckwith JG, Flashman LA, Paulsen K and Greenwald RM: Maximum principal strain and strain rate associated with concussion diagnosis correlates with changes in corpus callosum white matter indices. Ann Biomed Eng 40: 127-140, 2012.

11. Eierud C, Craddock RC, Fletcher S, Aulakh M, King-Casas B, Kuehl D and LaConte SM: Neuroimaging after mild traumatic brain injury: Review and meta-analysis. Neuroimage Clin 4: 283-294, 2014.

12. Bramlett HM, Kraydieh S, Green EJ and Dietrich WD: Temporal and regional patterns of axonal damage following traumatic brain injury: A beta-amyloid precursor protein immunocytochemical study in rats. J Neuropathol Exp Neurol 56: 1132-1141, 1997.

13. Paxinos G and Watson C: The Rat Brain in Stereotaxic Coordinates. Elsevier Publishing, Amsterdam, pp82-100, 1997.

14. Kilbourne M, Kuehn R, Tosun C, Caridi J, Keledjian K, Bochicchio G, Scalea T, Gerzanich V and Simard JM: Novel model of frontal impact closed head injury in the rat. J Neurotrauma 26: 2233-2243, 2009.

15. Fehily B and Fitzgerald M: Repeated mild traumatic brain injury: Potential mechanisms of damage. Cell Transplant 26: 1131-1155, 2017.

16. Xiang L, Zhang YT, Liang P, Wei H, Peng LL and Li LS: Value of $\beta$-APP and NF-L as markers in evaluation of rat diffuse axonal injury. J Third Military Med Univ 37: 2255-2260, 2015.

17. Browne KD, Chen XH, Meaney DF and Smith DH: Mild traumatic brain injury and diffuse axonal injury in swine. J Neurotrauma 28: 1747-1755, 2011.

18. Gasparetto EL, Rueda Lopes FC, Domingues RC and Domingues RC: Diffusion imaging in traumatic brain injury. Neuroimaging Clin N Am 21: 115-125, 2011.

19. McCrory P, Meeuwisse WH, Aubry M, Cantu B, Dvorák J, Echemendia RJ, Engebretsen L, Johnston K, Kutcher JS, Raftery M, et al: Consensus statement on concussion in sport: The 4th International Conference on Concussion in Sport held in Zurich, November 2012. Br J Sports Med 47: 250-258, 2013.

20. Ham TE and Sharp DJ: How can investigation of network function inform rehabilitation after traumatic brain injury. Curr Opin Neurol 25: 662-669, 2012.

21. Lipton ML, Gellella E, Lo C, Gold, T, Ardekani BA, Shifteh K, Bello JA and Branch CA: Multifocal white matter ultrastructural abnormalities in mild traumatic brain injury with cognitive disability: A voxel-wise analysis of diffusion tensor imaging. J Neurotrauma 25: 1335-1342, 2008.

22. Wang Y, Wang Q, Haldar JP, Yeh FC, Xie M, Sun P, Tu TW, Trinkaus K, Klein RS, Cross AH and Song SK: Quantification of increased cellularity during inflammatory demyelination. Brain 134: 3590-601, 2011. 